\title{
Ville Korpela \\ Social Choice Theory: A Neglected Path to Possibility
}

\section{Aboa Centre for Economics}

Discussion paper No. 110

Turku 2016

The Aboa Centre for Economics is a joint initiative of the economics departments of the University of Turku and Åbo Akademi University.

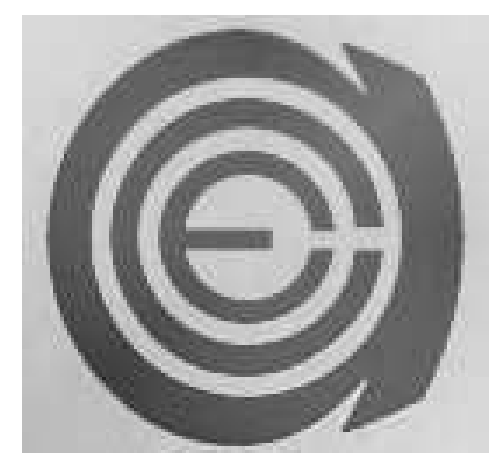


Copyright (C) Author(s)

ISSN 1796-3133

Printed in Uniprint Turku 2016 


\title{
Ville Korpela \\ Social Choice Theory: A Neglected Path to Possibility
}

\author{
Aboa Centre for Economics \\ Discussion paper No. 110 \\ October 2016 (first draft May 2016)
}

\begin{abstract}
Often preferences of agents are such that any sensible goal of the collective must admit a tie between all alternatives. A case in point is the Condorcet cycle with 3 alternatives and 3 voter. The standard formulation in mechanism design stipulates that in this case all alternatives must be equilibrium outcomes of the decision making mechanism. However, as far as the idea of an equilibrium is to predict the outcome of a mechanism, we could just as well demand that there are no equilibria at all. Although this may seem innocent, and in a technical sense that's right, it allows the mechanism designer to achieve goals that are otherwise impossible to implement.
\end{abstract}

JEL Classification: C72; D71

Keywords: Condorcet Criterion; Collective Decision Making; Implementation; Impossibility Result; Nash Equilibrium; Social Choice Theory 


\section{Contact information}

Ville Korpela

Department of Economics

University of Turku

FI-20014, Finland

Email: ville.korpela (at) utu.fi

\section{Acknowledgements}

I thank Arunava Sen for comments that have greatly improved this paper and Academy of Finland for financial support. 


\section{Introduction}

The fact that social choice theory was born in the aftermath of Arrow's impossibility theorem (Arrow [4]) was an omen for things to come: Results in this field have had a negative connotation ever since, either saying that no goal of society can satisfy certain desiderata $([\mathbf{4}],[\mathbf{2 4}],[\mathbf{5 7}])$, or that there would be no reliable way to collect the information that is needed anyway $([\mathbf{9}],[\mathbf{1 0}],[\mathbf{1 5}],[\mathbf{1 9}],[\mathbf{2 6}],[\mathbf{2 7}],[\mathbf{2 8}],[\mathbf{3 2}],[\mathbf{5 4}],[\mathbf{5 5}])$. The second problem is more fundamental in the sense that, at the end of the day, a decision needs to be made.

To be precise, and to define concepts we need later on, let $N=\{1, \ldots, n\}$ be the set of individuals, $A$ the set of alternatives, $\Theta=\Theta_{1} \times \cdots \times \Theta_{n}$ the set of states, and $\geq_{i}^{\theta}$ the preference relation of individual $i$ over $A$ at state $\theta$. Furthermore, suppose that the state space is unrestricted 1 and the goal of the collective, or the choice rule, can be represented as a mapping $f: \Theta \rightarrow A$ that associates a desired alternative $f(\theta) \in A$ to each state $\theta \in \Theta$. Then, either some individual $i$ wants to misrepresent his or her information at some state $\theta=\left(\theta_{1}, \theta_{2}, \ldots, \theta_{n}\right)$, that is

$$
f\left(\theta_{i}^{\prime}, \theta_{-i}\right)>_{i}^{\theta} f\left(\theta_{i}, \theta_{-i}\right) \text { for some } \theta_{i}^{\prime} \in \Theta_{i},
$$

$f$ is dictatorial (selects the best alternative of some individual at all states), or it has only 2 alternatives in the range $|f(\Theta)|=2 \cdot 2$ This is the famous Gibbard-Satterthwaite -theorem $([\mathbf{2 7}],[\mathbf{5 5}])$ and a choice rule that is not prone to this type of misrepresentation is called strategy-proof.

Two potential ways to escape this impossibility suggest themselves immediately. The outcome could be random, that is $f(\theta) \in \Delta(A)$, or it could be a subset of alternatives, that is $f(\theta) \subseteq A$. Unfortunately, both generalizations arrive at a similar conclusion as the GS -theorem. In the first case dictatorship is just replaced with random dictatorship $([\mathbf{2 6}])$, and in the second case, a similar conclusion holds for all sensible ways to generalize misrepresentation to set valued functions i.e. correspondences $([\mathbf{9}],[\mathbf{1 0}],[\mathbf{1 5}],[\mathbf{1 9}])$.

After the birth of mechanism design roughly in the late 1960s and early 1970s, pio-

\footnotetext{
${ }^{1}$ By unrestricted state space we mean that for any preference profile $\geq=\left(\geq_{1}, \geq_{2}, \ldots, \geq_{n}\right)$, there exists a state $\theta \in \Theta$, such that $\geq_{i}^{\theta}=\geq_{i}$ for all $i \in N$. In words, all preference configurations are possible.

${ }^{2}$ As usual, $\theta_{-i}$ is the profile $\left(\theta_{1}, \ldots, \theta_{i-1}, \theta_{i+1}, \ldots, \theta_{n}\right)$ that specifies the preference relation of each individual except $i$, and $f(\Theta)$ is the set $\{f(x) \mid x \in A\}$.
} 
neered by Leonid Hurwicz, Stanley Reiter and Eric Maskin, new possibilities began to emerge $3^{3}$ Unfortunately, in the case of unrestricted state space, this approach has not lead that far. Nowadays we know that if the Nash equilibrium correspondence of a decision mechanism is a function, then it is either dictatorial, constant, or selects between two alternative only, and in all other cases, it tends to be too large in the sense of selecting too many alternatives at each state 4 On the other hand, while some refinements of Nash equilibrium admittedly give more permissive results, like virtual implementation $([\mathbf{2}])$, subgame perfect implementation $([\mathbf{3}],[\mathbf{3 8}],[\mathbf{5 9}])$ and implementation using undominated strategies $([\mathbf{4 9}])$, they all have well-known problems, and moreover, Nash equilibrium is certainly the most natural solution concept since it demands the least amount of cognitive power from the individuals 5

In retrospect it seem that about the only way to realize a choice rule with good properties is to identify logical connections in the set of preferences - Black's single-peaked domain (Black [12]) with the median voter rule is a case in point $([\mathbf{1 3}],[\mathbf{4 0}])$.6 This is often unsatisfactory, and utterly so as a general solution, since there is nothing to guarantee that such a logic will suggest itself or even be there. Although the common explanation that preferences of voters are single-peaked over the left-right -axis is intuitively compelling, is there any strong reason why voter would conceptualize things like this, or is it rather so that in most voting situations a natural assumption is that the state space is unrestricted. Of course, if we admit this, then we have to admit that social choice theory is really facing an inescapable impossibility. We argue that this conclusion is far too hasty. In the standard formulation of mechanism design, if all alternatives are equally good for the collective, then all of them must be Nash equilibrium outcomes of the decision mechanism. However, since we do not need to predict the outcome in this particular case, we could just as well require that there are no equilibria at all. It may seem like there is no way this can have substantial consequences, but it does, and even in the unrestricted state space.

The rest of the paper is organized in the following way. In Sect. 2 we propose a modification to the standard mechanism design problem that is more in line with the interpretation of an equilibrium as a prediction. Although we do not want to rename old concept, but since it serves us so well here, we call the standard formulation

\footnotetext{
${ }^{3}$ For a review of the main contributions see $[\mathbf{8}],[\mathbf{1 6}],[\mathbf{1 7}],[\mathbf{3 1}],[\mathbf{3 4}],[\mathbf{3 6}],[\mathbf{4 8}]$ and $[\mathbf{5 8}]$.

${ }^{4}$ See Saijo [53] in addition.

${ }^{5}$ See Aghion, Fudenberg, Holden, Kunimoto and Tercieux (2015): "Subgame-Perfect Implementation Under Information Perturbations". The Quarterly Journal of Economics 127(4): 1843-1881.

${ }^{6}$ Another one is a quasi-linear environment with the VCG -mechanism. See also Aswal et al. [7].
} 
resolute mechanism design and the new formulation irresolute mechanism design. Then, in Sect. 3, we present some general results. Sect. 4 shows that our modification expands the set of Nash implementable choice rules even in the unrestricted state space. In particular, a specific Condorcet extension is now implementable in the case of 3 individuals and 3 alternatives, something that was certainly not possible in the standard sense. Sect. 5 concludes with a short discussion.

\section{The Devil is in the Details: Resolute vs. Irresolute Mechanism Design}

Leonid Hurwicz $([\mathbf{2 9}],[\mathbf{3 0}])$ was the first to give an explicit formulation of the idea that the goal of society can be separated from the mechanism that is used to realize it.7 Given $n$ message spaces $M_{1}, \ldots, M_{n}$, one for each individual, a mechanism $g$ on $A$ is a mapping

$$
g: M_{1} \times \cdots \times M_{n} \rightarrow A .
$$

We denote $M=M_{1} \times \cdots \times M_{n}=\stackrel{x}{i=1}^{n} M_{i}$, and write this mechanism as $G=(M, g) \cdot 8$ In contrast to strategy-proofness, where the only concern is whether individuals have an incentive to lie or not, we need to be more exact on how we expect them to behave. The most natural assumption, and the one that was used at the very beginning, is that a Nash equilibrium will be played.

Naturally, whether a given message is a Nash equilibrium or not, will depend on the true state. Once a state $\theta \in \Theta$ has been given, and preferences are therefore fixed, mechanism $G$ becomes a game $\Gamma(\theta)=(G, \theta)$. The message profile $m^{*}=\left(m_{1}^{*}, \ldots, m_{n}^{*}\right)$ is a pure strategy Nash equilibrium of this game if, and only if, $g\left(m^{*}\right) \geq_{i}^{\theta} g\left(m_{i}, m_{-i}^{*}\right)$ for all $i \in I$ and all $m_{i} \in M_{i} 9^{9}$ The set of all pure strategy Nash equilibrium profiles of $\Gamma(\theta)$ is denoted by $N E(G, \theta)$. Now, with all this machinery in place, we can formulate what Hurwicz ment.

Definition 1 (Resolute Mechanism Design). Choice rule $f: \Theta \rightarrow A$ is Nash implementable by a resolute mechanism if there exists $G=(M ; g)$ such that $g(N E(G, \theta))=$ $f(\theta)$ for all $\theta \in \Theta$.

\footnotetext{
${ }^{7}$ Historical details can be found from Jackson [31], Maskin and Sjöström [34] and Moore [36].

${ }^{8}$ Although $g$ already defines the message space.

${ }^{9}$ Here $m_{-i}^{*}=\left(m_{1}^{*}, \ldots, m_{i-1}^{*}, m_{i+1}^{*}, \ldots, m_{n}^{*}\right)$, and $\left(m_{i}, m_{-i}^{*}\right)=\left(m_{1}^{*}, \ldots, m_{i-1}^{*}, m_{i}, m_{i+1}^{*}, \ldots, m_{n}^{*}\right)$, as usual.
} 
In words, exactly those alternatives that choice rule $f$ regards as optimal are Nash equilibrium outcomes of $G$ at all states. The path-braking result of Maskin [35] says that if a choice rule is Nash implementable, then it is (Maskin) monotonic, and if it is monotonic and satisfies no-veto power (NVP), then it is Nash implementable 10 Let

$$
L_{i}(x, \theta) \equiv\left\{y \in A \mid x \geq_{i}^{\theta} y\right\}
$$

be the lower contour set of $x$ for individual $i$ at state $\theta$. Choice rule $f$ is monotonic, if for all $\theta, \psi \in \Theta$, and all $x \in f(\theta)$, if $L_{i}(x, \theta) \subseteq L_{i}(x, \psi)$ for all $i \in I$, then $x \in f(\psi){ }^{11}$ It satisfies no-veto power, if for all $\theta \in \Theta$, and all $x \in A$, if $x$ is the best alternative of at least $n-1$ agents at state $\theta$, then $x \in f(\theta)$.

Although this approach helps, it does not get us far in the case of unrestricted domain. By Maskin [35] a choice rule that satisfies Definition 1 must be monotonic, and if it is single-valued as well, then the result of Muller and Satterthwaite [41] says that it must be strategy-proof. Therefore, by the GS -theorem, the choice rule must be either dictatorial, constant or select between two alternatives only. However, although correspondences do not help with strategy-proofness, they do now. Maskin [35] shows that the Pareto correspondence, which selects all Pareto optimal alternatives at each state, and also the individually rational correspondence, which for a fixed alternative, selects all those alternatives that are considered at least as good by all, are both Nash implementable by a resolute mechanism. Unfortunately, even so, we are still left with two well-know problem: (1) The set of alternatives that these correspondences regard as acceptable are too large (even a dictatorial rule is Pareto optimal) and (2) once the mechanism has multiple equilibria at each states this will almost certainly lead to a coordination failure (what is the equilibrium that one anticipates others to play).

As we already explained in the introduction, although some refinements of Nash equilibrium give more optimistic results, it is a big question mark whether these results are very practical. However, even if they are, it is extremely unsatisfactory that the most natural solution concept, that of Nash equilibrium, does not give very encouraging results in the unrestricted state space.

We propose a novel approach to overcome some of these difficulties. To introduce the idea, suppose that $N=\{1,2,3\}, A=\{a, b, c\}$, and preferences at state $\theta$ are as in the

\footnotetext{
${ }^{10} \mathrm{~A}$ full characterization (a necessary and sufficient condition) was later given by Moore and Repullo [37].

${ }^{11}$ Monotonicity is called strong positive association by Muller and Satterthwaite (1977).
} 
table below 12

\begin{tabular}{ccc} 
Individual 1 & Individual 2 & Individual 3 \\
\hline$a$ & $c$ & $b$ \\
$b$ & $a$ & $c$ \\
$c$ & $b$ & $a$
\end{tabular}

TABLE 1. A Condorcet cycle

These preferences exhibit what is known as a Condorcet cycle $([\mathbf{2 3}],[\mathbf{6 1}])$ - but this is not the point. The point is that while it is natural to insist that choice rule $f$ selects all alternatives at state $\theta$, that is $f(\theta)=A$, it is not equally compelling to insist that $g(N E(G, \theta))=A$ as in Definition 1. We could just as well be satisfied with $g(N E(G, \theta))=\emptyset($ that is $N E(G, \theta)=\emptyset){ }^{13}$ After all, if the mechanism designer does not care what is the final outcome, what difference does it make if the mechanism does not have an equilibrium? This is even more so if the mechanism treats all alternatives in equal manner.

OBSERVATION: As far as the idea of an equilibrium is to predict the outcome of a mechanism, there is clearly no need for a decision making mechanism to always have an equilibrium. $\diamond$

Whether this is the purview of game theory community in general $([\mathbf{2 5}],[\mathbf{3 3}],[\mathbf{4 2}],[\mathbf{4 5}]$, $[\mathbf{5 6}])$, and opinions to the opposite have certainly been presented $([\mathbf{6}],[\mathbf{1 1}],[\mathbf{5 0}])$, it is clear that without this interpretation the enterprise of mechanism design would be pretty much void $([\mathbf{8}],[\mathbf{1 6}],[\mathbf{1 7}],[\mathbf{3 1}],[\mathbf{3 4}],[\mathbf{3 6}],[\mathbf{4 8}],[\mathbf{5 8}])$. Despite of what the commonly accepted view is, this observation does suggest that a certain amount of slack is possible in Definition 1.

Definition 2 (Irresolute Mechanism Design). Choice rule $f: \Theta \rightarrow A$ is Nash implementable by an irresolute mechanism if there exists a mechanism $G=(M ; g)$, such that $(\mathbf{1}) g(N E(G, \theta))=f(\theta)$ whenever $f(\theta) \neq g(M)$ and (2) either $g(N E(G, \theta))=$ $f(\theta)$ or $g(N E(G, \theta))=\emptyset$ if $f(\theta)=g(M)$.

This definition goes directly against an old tradition in social choice theory that consider consistency as an important property of a mechanism $([\mathbf{1}],[\mathbf{1 8}],[\mathbf{2 1}],[\mathbf{2 2}],[\mathbf{4 6}]$,

\footnotetext{
${ }^{12}$ The convention is that an alternative higher in the table is preferred.

${ }^{13}$ This requires that infinite message spaces are allowed, otherwise there would exist at least one mixed-strategy equilibrium $([\mathbf{4 3}],[\mathbf{4 4}])$. For all practical purposes, however, this may only require that individuals see the message space as potentially infinite (which could be generated by waiting time for example). See also Artemov [5].
} 
$[\mathbf{4 7}])^{14}$ On the other hand, since we violate this property in the weakest way imaginable, we should rather worry whether it make any difference at all. Two things indicate that it might. First of all, we know from the work of Donald Saari ([51],[52]) that a small set of preference configurations are behind most of the problems, and second, resent developments in mechanism design show that small things can have a huge effect ([14] is a case in point).

\section{General Results}

For a given CR $f: \Theta \rightarrow A$, define $\Theta^{R} \subseteq \Theta$ as the set

$$
\Theta^{R}=\{\theta \in \Theta \mid f(\theta) \neq A\} .
$$

In words, this is the set of states where all alternatives are not considered equally good. Using essentially the same methods as Maskin [35] we get the following results.

Theorem 1. If CR $f: \Theta \rightarrow A$ is Nash implementable by an irresolute mechanism, then $f: \Theta^{R} \rightarrow A$ is monotonic.

Proof. Suppose $G=(M ; g)$ is an irresolute mechanism that Nash implements $f$. Since for all $\theta \in \Theta^{R}$ and all $a \in f(\theta)$, there is an equilibrium $m^{*} \in N E(G, \theta)$ such that $g\left(m^{*}\right)=a$, the claim follows directly from Maskin $[\mathbf{3 5}]$

For the converse additional conditions are needed.

Definition 3. We say that CR $f: \Theta^{R} \rightarrow A$ is irresolute symmetric, if for all $\theta \in \Theta \backslash \Theta^{R}$, either (i) there does not exist any $\psi \in \Theta^{R}$ and $x \in f(\psi)$, such that $L_{i}(x, \psi) \subseteq L_{i}(x, \theta)$ for all $i \in N$, or (ii) for any $x \in A$, there exists $\psi \in \Theta^{R}$ such that $x \in f(\psi)$, and $L_{i}(x, \psi) \subseteq L_{i}(x, \theta)$ for all $i \in N$.

Definition 4. We say that CR $f: \Theta \rightarrow A$ satisfies strict no-veto power (SNPV), if for all $\theta \in \Theta$, and all $x \in A$, if $x$ is the top alternative of at least $n-1$ agents at state $\theta$, then $f(\theta)=\{x\}$.

Notice that NVP requires only that under these conditions $x \in f(\theta)$ is the case.

Theorem 2. If CR $f: \Theta^{R} \rightarrow A$ is monotonic, irresolute symmetric, and satisfies SNVP, then CR $f: \Theta \rightarrow A$ is Nash implementable by an irresolute mechanism.

\footnotetext{
${ }^{14}$ Consistency means that at least one equilibrium must exist at all states.
} 
Proof. We use a modification of the Maskin-mechanism (see [35]) to prove this claim. Let the message space of agent $i$ be $M_{i}=\Theta^{R} \times A \times \mathbb{N}_{+}$, denote a typical message of agent $i$ by $m_{i}=\left(\theta^{i}, x^{i}, n^{i}\right)$, and define the outcome function $g: M \rightarrow A$ by the following three rules:

(1) If $m_{i}=\left(\theta, x, n^{i}\right)$ for all $i \in N$, and $\{x\}=f(\theta)$, then $g(m)=x$.

(2) If $m_{j}=\left(\theta, x, n^{j}\right)$ for all $j \in N \backslash\{i\}, m_{i}=\left(\theta^{i}, x^{i}, n^{i}\right)$, and $\{x\}=f(\theta)$, then

$$
g(m)= \begin{cases}x^{i}, & \text { if } x^{i} \in L_{i}(x, \theta) \\ x, & \text { otherwise }\end{cases}
$$

(3) In all other cases, denote $k=\underset{i \in N}{\operatorname{argmax}} n^{i}$, and set

$$
g(m)=x^{k}
$$

Let us verify that $G=(M ; g)$ implements $f: \Theta \rightarrow A$. First of all, since SNPV implies NPV, we know by Maskin [35] that $G$ Nash implements $f: \Theta^{R} \rightarrow A$. Therefore, we only need to consider states in $\Theta \backslash \Theta^{R}$.

Suppose that $\psi \in \Theta \backslash \Theta^{R}$. If mechanism $G$ has a Nash equilibrium under rule (1), then all alternatives in $A$ must be Nash equilibrium outcomes under rule (1) due to the fact that $f: \Theta^{R} \rightarrow A$ is irresolute symmetric. Therefore, in this case, Definition 2 is satisfied. Assume, then, that there is a Nash equilibrium under rule (2) or (3). By SNVP this means that $f(\psi)$ is a singleton, which is a contradiction, since $\psi \in \Theta \backslash \Theta^{R}$ means that $f(\psi)=A$. Taken together these two observations prove our claim.

\section{Just How Deep Does the Rabbit Hole Go?}

Since our sufficient condition in Theorem 2 is fairly technical, we give an example to shows that Definition 2 really makes a difference. Suppose that there are three individuals $N=\{1,2,3\}$, three alternatives to choose form $A=\{a, b, c\}$, and all profiles of strict orderings are possible. Alternative $x \in A$ is a Condorcet winner at state $\theta$ if it beats all other alternatives in a pairwise comparison 15 Define choice rule $f^{\text {Con }}: \Theta \rightarrow A$ by the rule:

$$
f^{\operatorname{Con}}(\theta)= \begin{cases}x, & \text { if } x \text { is a Condorcet winner at } \theta, \\ A, & \text { otherwise. }\end{cases}
$$

\footnotetext{
${ }^{15}$ At least two individuals prefer $x$ to $y$ for both $y \in A \backslash\{x\}$.
} 
In the literature $f^{C o n}$ is called a Condorcet extension $([\mathbf{2 3}],[\mathbf{6 1}])$. Furthermore, in this simple case of 3 individuals and 3 alternatives, most, if not all, reasonable Condorcet extension coincide with $f^{\text {Con }}$ (the top cycle for example).

Since exactly all strict rankings are possible, there are $6^{3}=216$ preference profiles in the domain of $f^{\text {Con }}$, only 12 of which do not have a Condorcet winner. In fact, $f^{C o n}$ is the closest thing to a function that one can hope for in this domain without violating either anonymity or neutrality 16

Lemma 1. $f^{\text {Con }}: \Theta \rightarrow A$ is not Nash implementable by a resolute mechanism (or in the standard sense).

Proof. This follows from the result of Maskin [35] once we have shown that $f^{\text {Con }}$ is not monotonic. Suppose that at state $\theta$ preferences are:

\begin{tabular}{ccc} 
Individual 1 & Individual 2 & Individual 3 \\
\hline$a$ & $c$ & $b$ \\
$b$ & $a$ & $c$ \\
$c$ & $b$ & $a$
\end{tabular}

Thus $f^{\operatorname{Con}}(\theta)=A$ by definition. Suppose, then, that at state $\psi$ preferences are instead:

\begin{tabular}{ccc} 
Individual 1 & Individual 2 & Individual 3 \\
\hline$a$ & $c$ & $b$ \\
$b$ & $a$ & $a$ \\
$c$ & $b$ & $c$
\end{tabular}

We get these from the preferences at $\theta$ by propping $a$ above $c$ in the ranking of individual 3. Now $f^{C o n}(\psi)=\{a\}$ by definition. Therefore, $f^{C o n}$ is not monotonic, since monotonicity would imply that $b \in f^{\mathrm{Con}}(\psi)$, and as a consequence not Nash implementable by a resolute mechanism either.

Although this lemma does not tell us anything we did not already know, it is instructive for the things to come. Now consider the CR $f^{\text {Con }}: \Theta^{R} \rightarrow A$. As there are only 12 profiles where a unique Condorcet winner does not exist, $\Theta^{R}$ is almost as large as $\Theta$, namely $\left|\Theta^{R}\right|=216-12=204$ (or $100 \cdot \frac{204}{216} \approx 94 \%$ of the size).

Lemma 2. CR $f^{\text {Con }}: \Theta^{R} \rightarrow A$ is monotonic and satisfies SNVP.

\footnotetext{
${ }^{16}$ See the book of Moulin [39] for exact defintions.
} 
Proof. If at least two individuals think that alternative $x$ is the best at state $\theta$, then it must be a unique Condorcet winner, and therefore $x=f^{\operatorname{Con}}(\theta)$. Thus $f^{\operatorname{Con}}$ satisfies SNVP even if the domain is $\Theta$. The fact that Condorcet winner is always unique if the domain is $\Theta^{R}$ implies monotonicity. Suppose that $f^{\operatorname{Con}}(\theta)=\{x\}$. If alternative $x$ does not drop in the preference of anyone when going from state $\theta$ to state $\psi$, in the sense that $L_{i}(x, \theta) \subseteq L_{i}(x, \psi)$ for all $i \in N$, then it must beat the other two alternatives in a pairwise comparison also at state $\psi$. Hence $f^{\operatorname{Con}}(\psi)=\{x\}$ as required by monotonicity.

Taken together, Lemma 1 and 2 clearly indicate that those 12 preference profiles where Condorcet winner does not exist are behind most of the problem. But does Definition 2 help us here? We show that it does.

Lemma 3. CR $f^{\text {Con }}: \Theta^{R} \rightarrow A$ is irresolute symmetric.

Proof. Let $\theta \in \Theta \backslash \Theta^{R}$. We show that item (i) in Definition 3 must hold. Suppose that for some $\psi \in \Theta^{R}$, such that $x=f(\psi)$, we have $L_{i}(x, \psi) \subseteq L_{i}(x, \theta)$ for all $i \in N$. This is impossible since it would imply that $x$ is a unique Condorcet winner also at $\theta$ which we know is not the case as $\theta \in \Theta \backslash \Theta^{R}$.

Corollary. CR $f^{\text {Con }}: \Theta \rightarrow A$ is Nash implementable by an irresolute mechanism.

Proof. By Lemma 2 and 3 this follows from Theorem 2.

\section{Concluding Discussion}

The result that we have derived is not a trick. On the contrary, we claim that there is a shortcoming in the original definition (Definition 1), and that our reformulation (Definition 2) is more appropriate and deserves a further study. The reason why it expands the set of implementable choice rules substantially is simple. Each equilibrium in a decision making mechanism under one preference profile implies constraints on what can be selected at other preference profiles through monotonicity. When a choice rule judge all alternatives equally good, a large bundle of constraints is generated, some of which are necessarily strong: Those alternatives that are valued highly by some agents must be valued little by others. Otherwise there would be something wrong with the choice rule that deems all alternatives equally good.

Although our goal was not to uncover all consequences of Definition 2, but rather to show that they are substantial, it is fair to ask whether, and to what extent, our 
Theorem depends on the parameter of the problem (number of individuals and number of alternatives)? To have more structure in the set of preferences, suppose that $N=\{1,2,3\}$, and $A=\{a, b, c, d\}$, and let preferences be as in Table 2 below. Most reasonable choice rules would suggest that alternatives $a, b$ and $c$ should all be held equally good at this profile. On the other hand, since alternative $d$ is not acceptable, Definition 2 does not help us. However, is there any reason to expect that the mechanism we used would not work, after all, why would anyone suggest $d$. It seems inevitable that the outcome would belong to the set $\{a, b, c\}$. Suppose, then, that preferences are as in Table 3 below instead.

\begin{tabular}{ccc} 
Individual 1 & Individual 2 & Individual 3 \\
\hline$a$ & $c$ & $b$ \\
$b$ & $a$ & $c$ \\
$c$ & $b$ & $a$ \\
$d$ & $d$ & $d$
\end{tabular}

TABLE 2. Another instance of a Condorcet cycle Individual $1 \quad$ Individual $2 \quad$ Individual 3

\begin{tabular}{lll}
\hline$a$ & $c$ & $d$ \\
$b$ & $a$ & $b$ \\
$c$ & $b$ & $c$ \\
$d$ & $d$ & $a$
\end{tabular}

TABLE 3. Yet another instance of a Condorcet cycle

Again, alternatives $a, b$ and $c$ form a Condorcet cycle, but this time individual 3 might suggest $d$. Therefore, it is not clear whether our Theorem can be generalized, and if, then exactly how. Despite of this, however, it is nice to know that in comparison to the standard case (Definition 1) where the only sensible thing to do is to make a majority decision between two alternatives, exactly the same principle becomes Nash implementable for all practical purposes (Definition 2) also in the case of three alternatives if infinite message spaces are allowed. 


\section{References}

[1] Abdou, J. and Keiding, H. (1991): "Effectivity Functions in Social Choice". Kluwer Academic Publishers, Deventer, Netherlands.

[2] Abreu, D. and Sen, A. (1990): "Virtual Implementation in Nash Equilibrium". Econometrica 59: 997-1021.

[3] Abreu, D. and Sen, A. (1990): "Subgame Perfect Implementation: A Necessary and Almost Suffcient Condition". Journal of Economic Theory 50: 285-299.

[4] Arrow, K. J. (1963): Social Choice and Individual Values. John Wiley and Sons, New York.

[5] Artemov, G. (2015): "Time and Nash implementation". Games and Economic Behavior 91: 229-236.

[6] Aumann, R. (1987): "What Is Game Theory Trying to Accomplish?" in Frontiers of Economics, eds. K. J. Arrow and S. Honkapohja. Oxford: Blackwell: 287-324.

[7] Aswal, N., Chatterji, S. and Sen, A. (2003): "Dictatorial Domains". Economic Theory 22(1): 45-62.

[8] Baliga, S. and Sjöström, T. (2007): Mechanism Design: Recent Developments. The New Palgrave Dictionary of Economics, Second Edition, eds. L. Blume and S. Durlauf.

[9] Barberá, S., Dutta, B. and Sen, A. (2001): "Strategy-proof Social Choice Correspondences". Journal of Economic Theory 101(2): 374-394.

[10] Barberá, S. (1977): Manipulation of Social Decision Functions". Journal of Economic Theory 15(2): 266-278.

[11] Binmore, K. (1990): Essays on the Foundations of Game Theory. WileyBlackwell, Cambridge, USA.

[12] Black, D. (1948): "On the Rationale of Group Decision-Making". Journal of Political Economy 56: 23-34.

[13] Blin, J. M. and Satterthwaite, M. A. (1976): "Strategy-Proofness and Single-Peakedness". Public Choice 26(1): 51-58.

[14] Dutta, B. and Sen, A. (2012): "Nash implementation with partially honest individuals". Games and Economic Behavior 74: 154-169.

[15] Ching, S. and Zhou, L. (2002): Multi-Valued Strategy-Proof Social Choice Rules". Social Choice and Welfare 19: 569-580. 
[16] Chorchón, L. C. (2007): The Theory of Implementation: What did We Learn? Working Paper, Universitat Carlos III, Spain.

[17] Chorchón, L. C. (1996): The Theory of Implementation of Socially Optimal Decisions in Economics. Palgrave McMillan, USA.

[18] Danilov, V. I. and Sotskov, A. I. (2002): Social Choice Mechanisms. Studies in Economic Design, Springer-Verlag, Germany.

[19] Duggan, J. and Schwartz, T. (2000): "Strategic Manipulability Without Resoluteness or Shared Beliefs: Gibbard-Satterthwaite Generalized". Social Choice Welfare 17, 85-93.

[20] Dummett, M., and Farquharson, R. (1961): "Stability in Voting". Econometrica 29: 33-43.

[21] Dutta, B. (1984): "Effectivity Functions and Acceptable Game Forms". Econometrica 52: 1151-1166.

[22] Dutta, B. and Pattanaik, P. K. (1978): "On Nicely Consistent Voting Systems". Econometrica 46: 163-170.

[23] Fishburn, P. C. (1977): "Condorcet Social Choice Functions". SIAM Journal on Applied Mathematics 33(3): 469-489.

[24] Fishburn, P. C. (1973): The Theory of Social Choice. Princeton, New Jersey: Princeton University Press.

[25] Fudenberg, D. and Tirole, J. (2000): Game Theory. The MIT Press, USA.

[26] Gibbard, A. (1977): "Manipulation of Voting Schemes that Mix Voting with Chance". Econometrica 45: 665-681.

[27] Gibbard, A. (1973): "Manipulation of Voting Schemes: A General Result". Econometrica 41: 587-601.

[28] Gärdenfors, P. (1976): "Manipulation of Social Choice Functions". Journal of Economic Theory 13(2): 217-228

[29] Hurwicz, L. (1972): On Informationally Decentralized Systems. In Decision and Organization, eds. R. Radner and C. B. McGuire, North-Holland, Amsterdam: 297-336.

[30] Hurwicz, L. (1960): Optimality and Informational Efficiency in Resource Allocation Processes. In Mathematical Methods in the Social Sciences, eds. K. J. Arrow, S. Karlin and P. Suppes, Stanford University Press: 27-46.

[31] Jackson, M. O. (2001): "A Crash Course in Implementation Theory". Social Choice and Welfare 18: 655-708.

[32] Kelly, J. S.: (1977): "Strategy-Proofness and Social Choice Functions 
Without Single-Valuedness". Econometrica 45: 439-446.

[33] Luce, D. and Raiffa, H. (1957): Games and Decisions. New York, Wiley.

[34] Maskin, E. and Sjöström, T. (2002): Implementation Theory. Chapter 5 in Handbook of Social Choice and Welfare, eds. K. J. Arrow and A. K. Sen, Elsevier.

[35] Maskin, E. (1999): "Nash Equilibrium and Welfare Optimality". Review of Economic Studies 66: 23-38.

[36] Moore, J. (1992): Implementation, Contracts, and Renegotiation in Environments with Complete Information. In Advances in Economic Theory: Sixth World Congress Vol. I, ed. J.-J. Laffont. Cambridge, UK, Cambridge University Press.

[37] Moore, J. and Repullo, R. (1990): "Nash Implementation: A Full Characterization". Econometrica 58: 1083-1099.

[38] Moore, J. and Repullo, R. (1988): "Subgame Perfect Implementation". Econometrica 56: 1191-1220.

[39] Moulin, H. (1988): Axioms of Cooperative Decision Making. Cambridge University Press, USA.

[40] Moulin, H. (1980): "On Strategy-Proofness and Single Peakedness". Public Choice 35(4): 437-455.

[41] Muller, E. and Satterthwaite, M. (1977): "The Equivalence of Strong Positive Association and Strategy-Proofness". Journal of Economic Theory 14: 412-418.

[42] Myerson, R. (1991): Game Theory: Analysis of Conflict. Harvard University Press, USA.

[43] Nash, J. (1950): "Equilibrium points in n-person games". Proceedings of the National Academy of Sciences 36(1): 48-49.

[44] Nash, J. (1951): "Non-cooperative games". The Annals of Mathematics 54(2): 286-295.

[45] Osborne, M. and Rubinstein, A. (1994): A Course in Game Theory. The MIT Press, Cambridge, England.

[46] Peleg, B. (1984): Game Theoretic Analysis of Voting in Committees. Cambridge University Press, Cambridge.

[47] Peleg, B. (1978): "Consistent Voting Systems". Econometrica 46(1): 153-161.

[48] Palfrey, T. (1992): Implementation in Bayesian Equilibrium: the Multiple 
Equilibrium Problem in Mechanism Design. In Advances in Economic Theory: Sixth World Congress Vol. I, ed. J.-J. Laffont. Cambridge, UK, Cambridge University Press.

[49] Palfrey, T. and Srivastava, S. (1991): "Nash Implementation Using Undominated Strategies". Econometrica 59: 479-501.

[50] Rubinstein, A. (1991): "Comments on the Interpretation of Game Theory". Econometrica 59(4): 909-924.

[51] Saari, D. G. (2001): Decisions and Elections, Explaining the Unexpected. Cambridge University Press, New York.

[52] Saari, D. G. (1995): Basic Geometry of Voting. Springer-Verlag, Germany.

[53] Saijo, T. (1987): "On Constant Maskin Monotonic Social Choice Functions". Journal of Economic Theory 42(2): 382-386.

[54] Sato, S. (2008): On Strategy-Proof Social Choice Correspondences". Social Choice and Welfare 31: 331-343.

[55] Satterthwaite, M. A. (1975): "Strategy proofness and Arrow's Conditions: Existence and Correspondence Theorems for Voting Procedures and Social Welfare Functions". Journal of Economic Theory 10: 187-217.

[56] Schelling, T. (1960): The Strategy of Conflict. Harvard University Press, Cambridge.

[57] Sen, A. K. (1970): Collective Choice and Social Welfare. Edinburgh: Oliver and Boyd.

[58] Serrano, R. (2004): "The Theory of Implementation of Social Choice Rules". SIAM Review 46(3): 377-414.

[59] Vartiainen, H. (2007): "Subgame Perfect Implementation: A Full Characterization". Journal of Economic Theory 133: 111-126.

[60] William, V. (1960): "Utility, Strategy and Social Decision Rules". Quarterly Journal of Economics 74: 507-535.

[61] Young, P. H. (1988): "Condorcet's Theory of Voting". The American Political Science Review 82(4): 1231-1244. 
The Aboa Centre for Economics (ACE) is a joint initiative of the economics departments of the Turku School of Economics at the University of Turku and the School of Business and Economics at Åbo Akademi University. ACE was founded in 1998. The aim of the Centre is to coordinate research and education related to economics.

Contact information: Aboa Centre for Economics, Department of Economics, Rehtorinpellonkatu 3, FI-20500 Turku, Finland.

www.ace-economics.fi

ISSN 1796-3133 